\title{
Variance Results for Closed-loop Identification Methods
}

\author{
Lennart Ljung and Urban Forssell \\ Department of Electrical Engineering \\ Linkping University, S-581 83 Linkping, Sweden \\ WWW: http://www.control.isy.liu.se \\ Email: ljung@isy.liu.se, ufo@isy.liu.se
}

1997-09-17

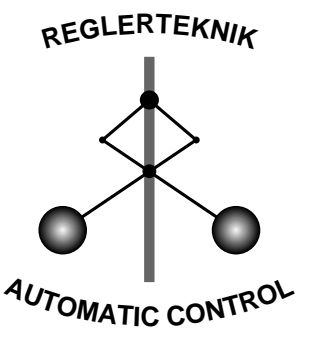

LINKÖPING

Report no.: LiTH-ISY-R-1976

Will be presented at CDC '97, San Diego.

Technical reports from the Automatic Control group in Linkping are available by anonymous ftp at the address 130.236.20.24 (ftp.control.isy.liu.se). This report is contained in the compressed postscript file 1976.ps.Z. 


\title{
Variance Results for Closed-loop Identification Methods
}

\author{
Lennart Ljung and Urban Forssell \\ Department of Electrical Engineering, Linköping University \\ S-581 83 Linköping, SWEDEN. \\ E-mail: ljung@isy.liu.se, ufo@isy.liu.se
}

\begin{abstract}
In this contribution we study the statistical properties of a number of closed-loop identification methods and parameterizations. A focus will be on asymptotic variance expressions for these methods and by studying the asymptotic variance for the parameter vector estimates we show that indirect methods fail to give better accuracy than the direct method.
\end{abstract}

\section{Introduction}

Currently there is a substantial interest in "Identification for Control" as is evident from the large number of conference sessions and journal papers devoted to this subject. The objective is to construct models that are suitable for high-performance control design and the use of closed-loop experiments has been a prominent feature in these approaches [2,13]. A main issue has been to construct methods that allow arbitrary shaping of the bias distribution in case of closed-loop data. In open loop this can be achieved by using an output error model with a fixed noise model/prefilter and applying a standard prediction error method. This approach breaks down in closed loop and the results will be biased. To circumvent this problem researchers have come up with various new identification methods/parameterizations, most of which have been derived in the prediction error framework (see, e.g., [1]).

From this perspective, it may seem as the interest in closed-loop issues in identification is quite new but in fact problems with closed-loop data have been studied since the early days of identification as can be seen from the survey paper [4] and the references therein. Several classical results on closed-loop identification can also be found in the text books [7] and [10].

The goal in this paper is to derive variance results for a number of closed-loop identification methods. As a vehicle for this we will use the standard prediction error theory described in [7].

\section{Preliminaries}

We will assume that the true system can be represented as

$$
\begin{aligned}
y(t) & =G_{0}(q) u(t)+v(t) \\
& =G_{0}(q) u(t)+H_{0}(q) e(t)
\end{aligned}
$$

Here $q$ is the forward shift operator and $\{e(t)\}$ is white noise with variance $\lambda_{0}$. The system is furthermore assumed to be controlled by a regulator

$$
u(t)=r(t)-F_{y}(q) y(t)
$$

The reference signal $\{r(t)\}$ is assumed independent of the noise $\{e(t)\}$ and we also assume that the regulator stabilizes the system and that either $G_{0}(q)$ or $F_{y}(q)$ contains a delay so that the closed-loop system is well defined.

We may write the closed-loop system as

$$
y(t)=G_{0}(q) S_{0}(q) r(t)+S_{0}(q) H_{0}(q) e(t)
$$

where $S_{0}(q)$ is the sensitivity function,

$$
S_{0}(q)=\frac{1}{1+F_{y}(q) G_{0}(q)}
$$

The input can be written as

$$
u(t)=S_{0}(q) r(t)-F_{y}(q) S_{0}(q) v(t)
$$

To reduce the notational burden we will from here on suppress the arguments $t, q, e^{i \omega}$, and $\omega$ whenever there is no risk of confusion.

The following definitions will also be convenient. The spectrum of the input is

$$
\Phi_{u}=\left|S_{0}\right|^{2} \Phi_{r}+\left|F_{y}\right|^{2}\left|S_{0}\right|^{2} \Phi_{v}
$$

where $\Phi_{r}$ is the spectrum of the reference signal and $\Phi_{v}=\left|H_{0}\right|^{2} \lambda_{0}$ the spectrum for the noise. We shall denote the two terms

$$
\Phi_{u}^{r}=\left|S_{0}\right|^{2} \Phi_{r}
$$

and

$$
\Phi_{u}^{e}=\left|F_{y}\right|^{2}\left|S_{0}\right|^{2} \Phi_{v}
$$

respectively. 
All methods studied in this paper are prediction error methods. We will assume that the parameter estimates $\hat{\theta}_{N}$ are computed as the minimizing argument of the least-squares criterion

$$
V_{N}(\theta)=\frac{1}{2 N} \sum_{t=1}^{N} \varepsilon^{2}(t, \theta)
$$

where $\varepsilon$ are the prediction errors.

The resulting estimates of the dynamics model and the noise model will in this paper be denoted $\hat{G}_{N}$ and $\hat{H}_{N}$,

$$
\hat{G}_{N}(q)=G\left(q, \hat{\theta}_{N}\right), \text { and } \hat{H}_{N}(q)=H\left(q, \hat{\theta}_{N}\right)
$$

For further details on prediction error identification we refer to [7].

\section{Approaches to Closed-loop Identification}

As mentioned in the introduction, closed-loop experiments are natural when the intended model use is control design. Other reasons for using closed-loop identification may be that the system is unstable, or that it has to be controlled for production economic and/or safety reasons. Sometimes the systems contain inherent feedback mechanisms which may not be known.

Depending on the assumptions made on the feedback mechanism the different closed-loop identification methods fall into the following main groups $[4,10]$ :

1. The direct approach: Ignore the feedback and apply a prediction error method directly using input and output measurements.

2. The indirect approach: First identify the closed-loop system, then compute an estimate of the open-loop system from the model obtained in the first step using the knowledge of the regulator.

3. The joint input-output approach: Model the input and output jointly as outputs from a system driven by the reference signal and noise and calculate an open-loop model from the identified joint system.

The direct approach gives consistency and optimal accuracy whenever the system can be correctly described in the model set (including the noise color). It can be applied to systems with arbitrary feedback mechanisms and requires no special software. Thus the direct approach should be regarded as the first choice of methods for closed-loop identification. A drawback with this approach is that we need good (parameterized) noise models for consistency. This fact should perhaps not be over-emphasized but, among other things, this implies that we can not use output error (or similar) model structures with fixed noise models to shape the bias distribution as in the case of open-loop data.

In the direct approach one typically works with models of the following kind:

$$
y(t)=G(q, \theta) u(t)+H(q, \theta) e(t)
$$

which will be assumed throughout the paper.

In the indirect approach the main focus is on correct modeling of the closed-loop system and consistency can be obtained even for incorrect noise models. These methods typically gives worse accuracy than the direct method, as we will see in this paper. They are also more complex than the simple direct method and require special software. It is also necessary that the external reference signal can be measured and that the regulator is correctly known. This last issue is particularly important since any error in the assumed regulator structure will translate into an error in the resulting open-loop model. Also any numerical errors in the computation of the open-loop model will affect the result. However, note that the second step in the indirect approach can be avoided if we use the following model, parameterized in terms of the open-loop parameters:

$$
y(t)=\frac{G(q, \theta)}{1+F_{y}(q) G(q, \theta)} r(t)+H_{c l}(q, \theta) e(t)
$$

This particular choice will be assumed in the following. The important issue is here that the resulting transfer function $\hat{G}_{N}$ will be the same regardless of the parameterization. Thus the parameterization may be important for algebraic and numerical reasons but does not affect the statistical properties of the estimates. Another interesting parameterization idea is used in the so-called dual-Youla method (see, e.g., [5, 6, 13]). The main advantage of this method is that the identified model is guaranteed to be stabilized by the controller used in the identification experiment. We stress that the dual-Youla method is a re-parameterization of the general indirect approach and hence all statistical properties are the same.

If the output $y$ and the input $u$ as well as the reference signal $r$ are available for measurements the joint input output approach is an alternative to the direct and indirect approaches. Note that in this approach no explicit knowledge of the feedback is required other than that it must be known/assumed to be of a certain (linear) structure. Several interesting methods that belong to this group have been presented $[12,14]$. These methods will not be further studied in this paper. A more comprehensive study of the different approaches can be found in [1]. 


\section{Variance of Transfer Function Estimates}

Let us now consider the asymptotic variance of the estimated transfer functions $\hat{G}_{N}$ and $\hat{H}_{N}$ using the Asymptotic Black-Box theory of Section 9.4 in [7].

Note that the basic result

$$
\operatorname{Cov}\left[\begin{array}{c}
\hat{G}_{N} \\
\hat{H}_{N}
\end{array}\right] \sim \frac{n}{N} \Phi_{v}\left[\begin{array}{cc}
\Phi_{u} & \Phi_{u e} \\
\Phi_{u e}^{*} & \lambda_{0}
\end{array}\right]^{-1}
$$

applies also to the closed-loop case. Here $n$ is the model order, $N$ the number of data, and $\Phi_{u e}$ the cross spectrum between input $u$ and noise source $e$ : $\Phi_{u e}=-F_{y} S_{0} H_{0} \lambda_{0}$

In particular, from this general expression we can solve for the upper left element:

$$
\operatorname{Cov} \hat{G}_{N} \sim \frac{n}{N} \frac{\Phi_{v}(\omega) \lambda_{0}}{\lambda_{0} \Phi_{u}-\left|\Phi_{u e}\right|^{2}}=\frac{n}{N} \frac{\Phi_{v}}{\left|S_{0}\right|^{2} \mid \Phi_{r}}=\frac{n}{N} \frac{\Phi_{v}}{\Phi_{u}^{r}}
$$

The result (12) - which also is the asymptotic CramerRao lower limit - shows that it is the signal-to-noise ratio $\Phi_{u}^{r} / \Phi_{v}$ that determines how well the open-loop transfer function can be estimated. The expression (12) also points to the basic problem in closed-loop identification: The purpose of feedback is to make the sensitivity function small, especially at frequencies with disturbances and poor system knowledge. Feedback will thus worsen the measured data's information about the system at these frequencies.

From the expression (12) it is clear that the part of the input that originates from the feedback has no information value when estimating $G$. Since this property is, so to say, inherent in the problem, it should come as no surprise that the result (12) applies also to other closed-loop identification methods, such as the general indirect method, the dual-Youla method and the coprime factor identification scheme. See $[3,1]$ for details.

It seems that since the result (11) is asymptotic in both the model order $n$ and the number of data $N$ :

$$
n \rightarrow \infty, N \rightarrow \infty, \frac{n}{N} \rightarrow 0
$$

the differences between the methods disappear. Thus it is impossible to rank the methods based on the accuracy of the transfer function estimates. Instead we turn to variance expressions for parameter estimates.

\section{Variance of Parameter Estimates: The Direct Approach}

Consider the model (9) and assume that the dynamics model and the noise model are independently parameterized,

$$
G(q, \theta)=G(q, \rho) \text { and } H(q, \theta)=H(q, \beta)
$$

where $\rho$ and $\beta$ refers to the following partitioning of the parameter vector $\theta$ :

$$
\theta=\left[\begin{array}{l}
\rho \\
\beta
\end{array}\right]
$$

Also assume that the true system is contained in the model set (in the sequel this will be denoted $\mathcal{S} \in \mathcal{M}$ ). Then, from the results in Section 9.3 in [7], we have the following: The covariance of the parameter estimate is

$$
\operatorname{Cov} \hat{\theta}_{N} \sim \frac{1}{N} P_{\theta}
$$

Here

$$
P_{\theta}=\lambda_{0} R_{\theta}^{-1}
$$

where $\lambda_{0}$ is the variance of the driving noise and

$$
R_{\theta}=\bar{E} \psi\left(t, \theta_{0}\right) \psi^{T}\left(t, \theta_{0}\right)
$$

We will in the following derive explicit expressions for $P_{\theta}$ in the case of a linear feedback regulator as in (2). The expressions will be given in the frequency domain. It will be convienient to consider the following augmented signal: Let

$$
\chi_{0}=\left[\begin{array}{l}
u \\
e
\end{array}\right]
$$

The spectrum of $\chi_{0}$ is

$$
\Phi_{\chi_{0}}=\left[\begin{array}{cc}
\Phi_{u} & \Phi_{u e} \\
\Phi_{u e}^{*} & \lambda_{0}
\end{array}\right]
$$

Since $\Phi_{u e}=-F_{y} S_{0} H_{0} \lambda_{0}$, we may also write this as

$$
\Phi_{\chi_{0}}=\Phi_{\chi_{0}}^{r}+\Phi_{\chi_{0}}^{e}
$$

where

$$
\Phi_{\chi_{0}}^{r}=\left[\begin{array}{cc}
\Phi_{u}^{r} & 0 \\
0 & 0
\end{array}\right] \text { and } \Phi_{\chi_{0}}^{e}=\lambda_{0}\left[\begin{array}{c}
F_{y} S_{0} H_{0} \\
-1
\end{array}\right]\left[\begin{array}{c}
F_{y} S_{0} H_{0} \\
-1
\end{array}\right]^{*}
$$

Using the frequency-domain results in Section 9.4 in [7] we see that $R_{\theta}$ can be written as

$$
R_{\theta}=\frac{1}{2 \pi} \int_{-\pi}^{\pi} \frac{1}{\left|H_{0}\right|^{2}} T_{\theta}^{\prime} \Phi_{\chi_{0}} T_{\theta}^{\prime *} d \omega
$$

where $T=\left[\begin{array}{ll}G & H\end{array}\right]$ and where $T_{\theta}^{\prime}=\left.\frac{d}{d \theta} T\right|_{\theta=\theta_{0}}$. From (19) it follows that

$$
T_{\theta}^{\prime} \Phi_{\chi_{0}} T_{\theta}^{\prime *}=T_{\theta}^{\prime} \Phi_{\chi_{0}}^{r} T_{\theta}^{\prime *}+T_{\theta}^{\prime} \Phi_{\chi_{0}}^{e} T_{\theta}^{\prime *}
$$

We may thus write

$$
R_{\theta}=R_{\theta}^{r}+R_{\theta}^{e}
$$


where $R_{\theta}^{r}$ is given by

$$
\begin{aligned}
R_{\theta}^{r} & =\frac{1}{2 \pi} \int_{-\pi}^{\pi} \frac{1}{\left|H_{0}\right|^{2}} T_{\theta}^{\prime} \Phi_{\chi_{0}}^{r} T_{\theta}^{\prime *} d \omega \\
& =\frac{1}{2 \pi} \int_{-\pi}^{\pi} \frac{\Phi_{u}^{r}}{\left|H_{0}\right|^{2}} G_{\theta}^{\prime} G_{\theta}^{\prime *} d \omega
\end{aligned}
$$

Note that $R_{\theta}^{r}$ only depends on $\Phi_{u}^{r}$ and not on the total input spectrum $\Phi_{u}$, as in the open-loop case. If we partition $R_{\theta}^{r}$ conformably with $\theta$ we see that, due to the chosen parameterization,

$$
R_{\theta}^{r}=\left[\begin{array}{cc}
R_{\rho}^{r} & 0 \\
0 & 0
\end{array}\right]
$$

where

$$
R_{\rho}^{r}=\frac{1}{2 \pi} \int_{-\pi}^{\pi} \frac{\Phi_{u}^{r}}{\left|H_{0}\right|^{2}} G_{\rho}^{\prime} G_{\rho}^{* *} d \omega
$$

Returning to $R_{\theta}^{e}$ we see that

$$
R_{\theta}^{e}=\frac{1}{2 \pi} \int_{-\pi}^{\pi} \frac{1}{\left|H_{0}\right|^{2}} T_{\theta}^{\prime} \Phi_{\chi_{0}}^{e} T_{\theta}^{\prime *} d \omega
$$

If we partition $R_{\theta}^{e}$ as

$$
R_{\theta}^{e}=\left[\begin{array}{cc}
R_{\rho}^{e} & R_{\rho \beta}^{e} \\
R_{\beta \rho}^{e} & R_{\beta}^{e}
\end{array}\right]
$$

we may write

$$
P_{\theta}=\lambda_{0}\left[\begin{array}{cc}
R_{\rho}^{r}+R_{\rho}^{e} & R_{\rho \beta}^{e} \\
R_{\beta \rho}^{e} & R_{\beta}^{e}
\end{array}\right]^{-1}
$$

The covariance of $\hat{\rho}_{N}$ is given by the top left block of $P_{\theta}$. It follows that

$$
\operatorname{Cov} \hat{\rho}_{N} \sim \frac{\lambda_{0}}{N}\left(R_{\rho}^{r}+\Delta\right)^{-1}
$$

where

$$
\Delta=R_{\rho}^{e}-R_{\rho \beta}^{e}\left(R_{\beta}^{e}\right)^{-1} R_{\beta \rho}^{e} \geq 0
$$

is the Schur complement of $R_{\beta}^{e}$ in the matrix $R_{\theta}^{e}$. Explicit expressions for $R_{\rho}^{e}, R_{\rho \beta}^{e}$ and $R_{\beta}^{e}$ can be derived using

$$
T_{\theta}^{\prime}\left[\begin{array}{c}
F_{y} S_{0} H_{0} \\
-1
\end{array}\right]=F_{y} S_{0} H_{0} G_{\theta}^{\prime}-H_{\theta}^{\prime}
$$

An important observation regarding the result (29) is that the term $\Delta$ is entirely due to the noise part of the input spectrum and since $\Delta \geq 0$ this contribution has a positive effect on the accuracy, contrarily to what one might have guessed. We conclude that in the direct method the noise in the loop is utilized in reducing the variance. Later we will see that for the indirect methods this contribution will be zero.

From (29) it is also clear that the worst-case experimental conditions - from the accuracy point of view
- is when there is no external reference signal present (i.e., when $\Phi_{r}=0$ ). In that case

$$
\operatorname{Cov} \hat{\rho}_{N} \sim \frac{\lambda_{0}}{N} \Delta^{-1}
$$

Thus $\Delta$ characterizes the lower limit of achievable accuracy for the direct method. Now, if $\Delta$ is nonsingular, we can consistently estimate the system parameters even though no reference signal is present. The exact conditions for this to happen are given in [8] for some common special cases. However, even if $\Delta$ is singular it will have a beneficial effect on the variance of the estimates, according to (29). Only when $\Delta=0$ there is no positive effect from the noise source on the accuracy of the estimates.

Let us study what makes $\Delta=0$ : According to (31), we can write

$$
\begin{aligned}
R_{\theta}^{e} & =E \psi_{e}\left(t, \theta_{0}\right) \psi_{e}^{T}\left(t, \theta_{0}\right) \\
\psi_{e}\left(t, \theta_{0}\right) & =\left[\begin{array}{c}
L(q) w(t) \\
-H_{\beta}^{\prime}(q) w(t)
\end{array}\right] \\
w(t) & =H_{0}^{-1}(q) e(t) \\
L(q) & =F_{y}(q) S_{0}(q) H_{0}(q) G_{\rho}^{\prime}\left(q, \theta_{0}\right)
\end{aligned}
$$

Here the number of rows in $L$ and $H_{\beta}^{\prime}$ are consistent with the partitioning (27). From well known leastsquares projections, we now recognize $\Delta$ as the error covariance matrix when estimating $L w$ from $H_{\beta}^{\prime} w$. If the noise model is very flexible, knowing $H_{\beta}^{\prime} w$ is equivalent to knowing all past $w$ (think, e.g., of $H$ being a FIR model of "almost infinite" length). Then $L w$ can be determined exactly from $H_{\beta}^{\prime} w$, and $\Delta=0$. At the other extreme, a fixed (and correct) noise model will make $\Delta=R_{\rho}^{e}=E L w(L w)^{T}$, which is the largest value $\Delta$ may have. This puts the finger on the value of information in the noise source $e$ for estimating the dynamics: It is the knowledge/assumption of a constrained noise model that improves the estimate of $G$.

\section{Variance of Parameter Estimates: The Indirect Approach}

We will now apply the same analysis tools as we used for the direct method in the previous section, to the indirect method.

Consider the following model (cf. (10))

$$
y(t)=\frac{G(q, \rho)}{1+F_{y}(q) G(q, \rho)} r(t)+H_{c l}(q, \beta) e(t)
$$

Estimating $\rho$ and $\beta$ in (34) is an open-loop problem. All standard open-loop results can thus be applied also in this case. As an example, assume $\mathcal{S} \in \mathcal{M}$, then we can immediately write down the expressions for the covariance of $\hat{\rho}$ :

$$
\operatorname{Cov} \hat{\rho}_{N} \sim \frac{\lambda_{0}}{N}\left(R_{c l}\right)^{-1}
$$


where

$$
R_{c l}=\frac{1}{2 \pi} \int_{-\pi}^{\pi} \frac{\Phi_{r}}{\left|H_{c l, 0}\right|^{2}} G_{c l, \rho}^{\prime} G_{c l, \rho}^{* *} d \omega
$$

Note that $G_{c l, \rho}^{\prime}=S_{0}^{2} G_{\rho}^{\prime}$ and $\left|H_{c l, 0}\right|^{2}=\left|S_{0}\right|^{2}\left|H_{0}\right|^{2}$, hence

$$
R_{c l}=\frac{1}{2 \pi} \int_{-\pi}^{\pi} \frac{\Phi_{u}^{r}}{\left|H_{0}\right|^{2}} G_{\rho}^{\prime} G_{\rho}^{\prime *} d \omega
$$

This is in fact identical to expression (25) and we conclude that, for the indirect method,

$$
\operatorname{Cov} \hat{\rho}_{N} \sim \frac{\lambda_{0}}{N}\left(R_{\rho}^{r}\right)^{-1}
$$

As we remarked before this covariance will always be larger than the covariance obtained in the direct method, equation (29). The difference stemming from the term $\Delta$ that is missing in (38). Thus, in terms of accuracy of the parameter estimates, the direct method outperforms the indirect.

\section{Indirect Identification with Optimal Accuracy}

In the previous section we saw that indirect identification gives worse accuracy than direct. This is not the case in general for indirect methods, as we will see presently. In the following we will review a result that was first derived in [9] (see also [4] and [11] for related results) namely that, under certain circumstances, indirect identification gives the same level of accuracy as direct identification.

\subsection{ARMAX Modeling}

Suppose we identify the closed-loop system using an ARMAX model

$$
A_{c l}(q) y(t)=B_{c l}(q) r(t)+C_{c l}(q) e(t)
$$

Thus, with $\eta$ denoting the closed-loop parameter vector, the system dynamics is modeled as

$$
G_{c l}(q, \eta)=\frac{B_{c l}(q)}{A_{c l}(q)}
$$

while the noise model becomes

$$
H_{c l}(q, \eta)=\frac{C_{c l}(q)}{A_{c l}(q)}
$$

Also assume $\mathcal{S} \in \mathcal{M}$. Next, let the regulator be given by

$$
F_{y}(q)=\frac{X(q)}{Y(q)}
$$

where the polynomials $X$ and $Y$ are assumed coprime. Then, if we in the second step of the indirect scheme model the open-loop system as

$$
A(q) y(t)=B(q) u(t)+C(q) e(t)
$$

we get the following. From

$$
G_{c l}=\frac{G}{1+G F_{y}}
$$

and

$$
H_{c l}=\frac{H}{1+G F_{y}}
$$

it follows that we may solve for $A, B$ and $C$ in

$$
\left\{\begin{array}{l}
A_{c l}=A Y+B X \\
B_{c l}=B Y \\
C_{c l}=C Y
\end{array}\right.
$$

Equation (46) may be interpreted as a system of linear equations in the open-loop parameters $\theta$,

$$
\Gamma \theta=\bar{\eta}
$$

where $\Gamma$ is completely determined by $X$ and $Y$ and where $\bar{\eta}$ depends on the estimated closed-loop parameters $\hat{\eta}$ and $Y$. The best unbiased estimate of $\theta$ is the Markov estimate,

$$
\hat{\theta}=\left[\Gamma^{T}(\operatorname{Cov} \bar{\eta})^{-1} \Gamma\right]^{-1} \Gamma^{T}(\operatorname{Cov} \bar{\eta})^{-1} \bar{\eta}
$$

which gives

$$
\operatorname{Cov} \hat{\theta}=\left[\Gamma^{T}(\operatorname{Cov} \bar{\eta})^{-1} \Gamma\right]^{-1}
$$

Now, by explicitly forming (47) and carrying out the algebra it can be shown that

$$
\operatorname{Cov} \hat{\theta} \sim \frac{\lambda_{0}}{N}\left[\bar{E} \psi\left(t, \theta_{0}\right) \psi\left(t, \theta_{0}\right)^{T}\right]^{-1}
$$

Here $\psi$ is the negative gradient of the open-loop prediction errors

$$
\varepsilon(t, \theta)=\frac{1}{C(q)}(A(q) y(t)-B(q) u(t))
$$

But (50) is equivalent to expression (14). Thus, under certain circumstances, indirect identification gives the same accuracy as direct identification. This result can be transformed to the frequency domain using expression $(20)$.

As will become more apparent below, the fact that the dynamics model and the noise model share the same poles is crucial for obtaining the same accuracy as with the direct approach.

\subsection{Independently Parameterized Noise Model} Suppose that we in the first step in the indirect method use the following model with an independently parameterized noise model:

$$
\begin{aligned}
y(t) & =G_{c l}\left(q, \rho_{\eta}\right) r(t)+H_{c l}\left(q, \beta_{\eta}\right) e(t) \\
& =\frac{B_{c l}(q)}{F_{c l}(q)} r(t)+\frac{C_{c l}(q)}{D_{c l}(q)} e(t)
\end{aligned}
$$


This is also known as a Box-Jenkins model. We assume also in this case that $\mathcal{S} \in \mathcal{M}$. Let the open-loop model be

$$
\begin{aligned}
y(t) & =G\left(q, \rho_{\theta}\right) u(t)+H\left(q, \beta_{\theta}\right) e(t) \\
& =\frac{B(q)}{F(q)} r(t)+\frac{C(q)}{D(q)} e(t)
\end{aligned}
$$

To find the indirect estimate of $G_{0}$ we may solve for $B$ and $F$ in (cf. (46))

$$
\left\{\begin{array}{l}
B_{c l}=B Y \\
F_{c l}=F Y+B X
\end{array}\right.
$$

This system of equations may be written in form similar to $(47)$ :

$$
\tilde{\Gamma} \rho_{\theta}=\bar{\rho}_{\eta}
$$

The Markov estimate is

$$
\hat{\rho}_{\theta}=\left[\tilde{\Gamma}^{T}\left(\operatorname{Cov} \bar{\rho}_{\eta}\right)^{-1} \tilde{\Gamma}\right]^{-1} \tilde{\Gamma}^{T}\left(\operatorname{Cov} \bar{\rho}_{\eta}\right)^{-1} \bar{\rho}_{\eta}
$$

This time, however, we do not obtain the open-loop expression for the variance. Instead it can be shown that

$$
\operatorname{Cov} \hat{\rho}_{\theta} \sim \frac{1}{N}\left(R_{\rho}^{r}\right)^{-1}
$$

where $R_{\rho}^{r}$ is given by (25). We once again conclude that, with an independently parameterized noise model, the indirect method gives worse accuracy than the direct method. The difference is quantified by the term $\Delta$ (cf. (29)) which is missing in (57). So by using an independently parameterized noise model in the indirect method the noise does not contribute in reducing the variance as in the direct method and the indirect method using ARMAX models.

\section{Summarizing Remarks}

We may summarize the main points of the paper as follows.

- A directly applied prediction error method will give consistency and optimal accuracy even with closed-loop data, given that the noise model can describe the true noise properties.

- In the direct approach the noise in the loop is utilized in reducing the variance. For indirect methods this contribution is zero unless the dynamics model and noise model share the same poles, as when ARMAX models are used.

- Use of a parameterized noise model will always lead to worse accuracy of the estimate $G$ compared to if a fixed (and correct) noise model is used. It is the knowledge/assumption of a constrained noise model that improves the estimate G.

\section{References}

[1] U. Forssell and L. Ljung. Closed-loop Identification Revisited. Technical Report LiTH-ISY-R-1959, Department of Electrical Engineering, Linköping University, Linköping, Sweden, 1997. Submitted to Automatica.

[2] M. Gevers. Towards a Joint Design of Identification and Control. In H. L. Trentelman and J. C. Willems, editors, Essays on Control: Perspectives in the Theory and its Applications, pages 111-151. Birkhäuser, 1993.

[3] M. Gevers, L. Ljung, and P. Van den Hof. Asymptotic variance expression for closed-loop identification and their relevance in identification for control. In Proceedings of the 11th IFAC Symposium on System Identification, volume 3, pages 1449-1454, Fukuoka,Japan, 1997.

[4] I. Gustavsson, L. Ljung, and T. Söderström. Identification of Processes in Closed Loop - Identifiability and Accuracy Aspects. Automatica, 13:59-75, 1977.

[5] F. R. Hansen. A fractional representation to closed-loop system identification and experiment design. Phd thesis, Stanford University, Stanford, CA, USA, 1989.

[6] F. R. Hansen, G. F. Franklin, and R. Kosut. Closed-loop identification via the fractional representation: experiment desgin. In Proceedings of the American Control Conference, pages 1422-1427, Pittsburg, PA, 1989.

[7] L. Ljung. System Identification: Theory for the User. Prentice-Hall, 1987.

[8] T. Söderström, I. Gustavsson, and L. Ljung. Identifiability conditions for linear systems operating in closed loop. Int. J. Control, 21(2):234-255, 1975.

[9] T. Söderström, L. Ljung, and I. Gustavsson. On The Accuracy Of Identification And the Design Of Identification Experiments. Technical Report 7428, Department of Automatic Control, Lund Institute of Technology, Lund, Sweden, 1974.

[10] T. Söderström and P. Stoica. System Identification. Prentice-Hall International, 1989.

[11] T. Söderström, P. Stoica, and B. Friedlander. An Indirect Prediction Error Method for System Identification. Automatica, 27:183-188, 1991.

[12] P. M. J. Van den Hof and R. J. P. Schrama. An Indirect Method for Transfer Function Estimation from Closed Loop Data. Automatica, 29(6):1523-1527, 1993.

[13] P. M. J. Van den Hof and R. J. P. Schrama. Identification and Control - Closed-loop Issues. Automatica, 31(12):1751-1770, 1995.

[14] P. M. J. Van den Hof, R. J. P. Schrama, R. A. de Callafon, and O. H. Bosgra. Identification of normalized coprime factors from closed-loop experimental data. European Journal of Control, 1(1):62-74, 1995. 